\title{
On the unsteady dynamics of synthetic leaves: Laboratory experiments using synchronized PIV and DIC
}

\author{
Lai Wing ${ }^{1}$, Dan Troolin ${ }^{1}$, Shyuan Cheng, ${ }^{2}$ Jiao Sun, ${ }^{2}$ and Leonardo P. Chamorro ${ }^{2}$ \\ 1 TSI Incorporated, USA. \\ ${ }^{2}$ Department of Mechanical Science and Engineering, University of Illinois, 61801, USA.
}

\begin{abstract}
The unsteady 3D dynamics of various synthetic leaves and the induced turbulence are systematically studied experimentally for representative Cauchy numbers in a wind tunnel under nearly uniform incoming flows. Synchronized digital image correlation (DIC) and high-frame-rate particle image velocimetry (PIV) are employed to track the structure dynamics simultaneously and the surrounding flow field to uncover the fluid-solid interaction. A high-resolution six-axis load cell is also used to quantify the synthetic leaves' induced force and torque under various flows. The shapes of synthetic leaves inspected are representative of selected environments (e.g., calm to windy weather; tropical to temperate climate). The Cauchy number is set to resemble those observed in natural conditions. This presentation will discuss insights from synchronized PIV-DIC techniques on the synthetic leaves' distinct behavior and wake flow response. Particular emphasis is placed on characterizing flow instability and the leave shape's role in the motions and force. For this purpose, we inspected the instantaneous force and torque as well as their structure. We will also discuss the relationship between leave shapes with force and torque fluctuations linking them with the leaf motion obtained from DIC measurements. In particular, the results show that selected leaf shapes experience significantly larger and distinct force and torque fluctuations and larger pitch magnitude, as shown in Fig. 5. A shared monotonically decreasing trend of the nondimensional frequency (Strouhal number, $S t=f L / U$ ) is evidenced for standard environmental conditions.
\end{abstract}

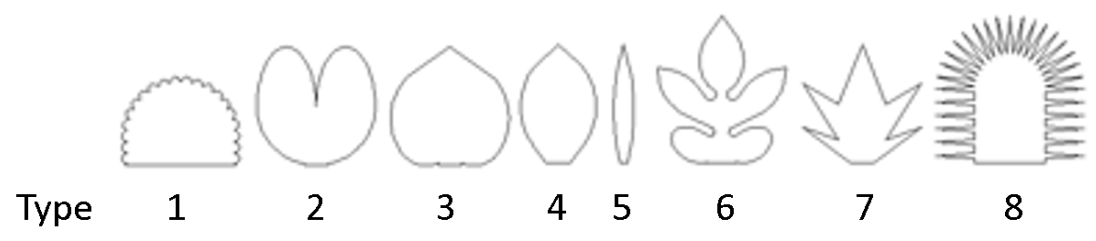

Figure 1. basic geometry of the synthetic leaf shapes.
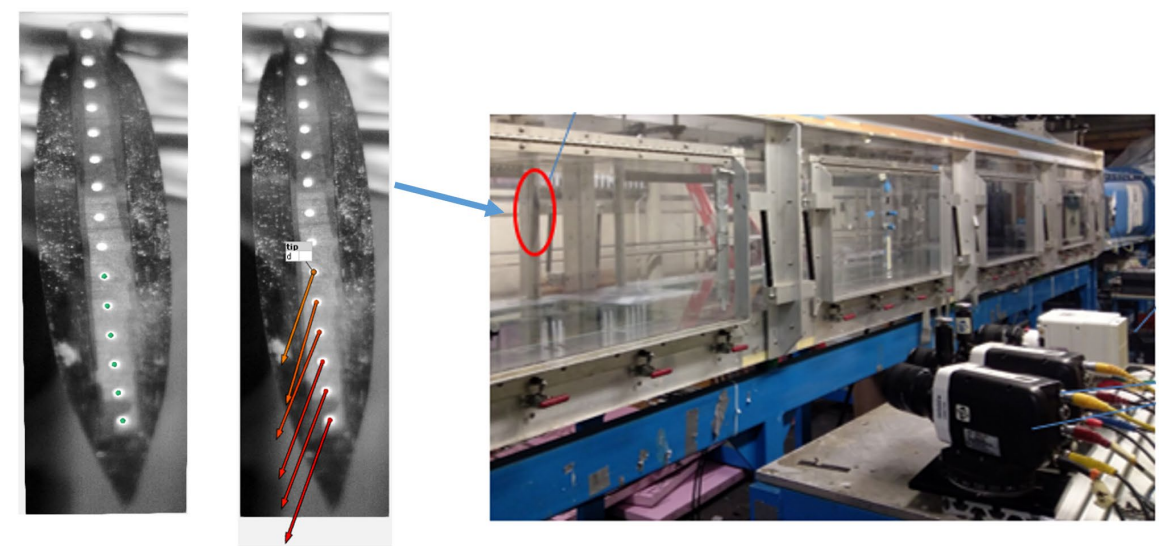

Figure 2. Photographies of the general setup illustrating a type-5 leaf geometry. 


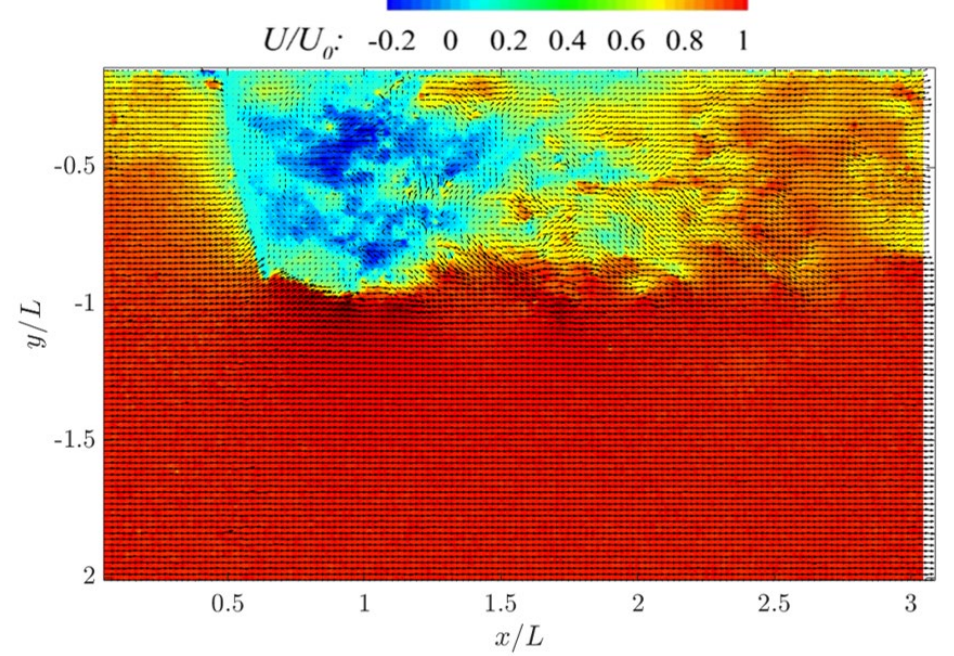

Figure 3. Example of an instantaneous velocity field in the near wake of a synthetic leaf.
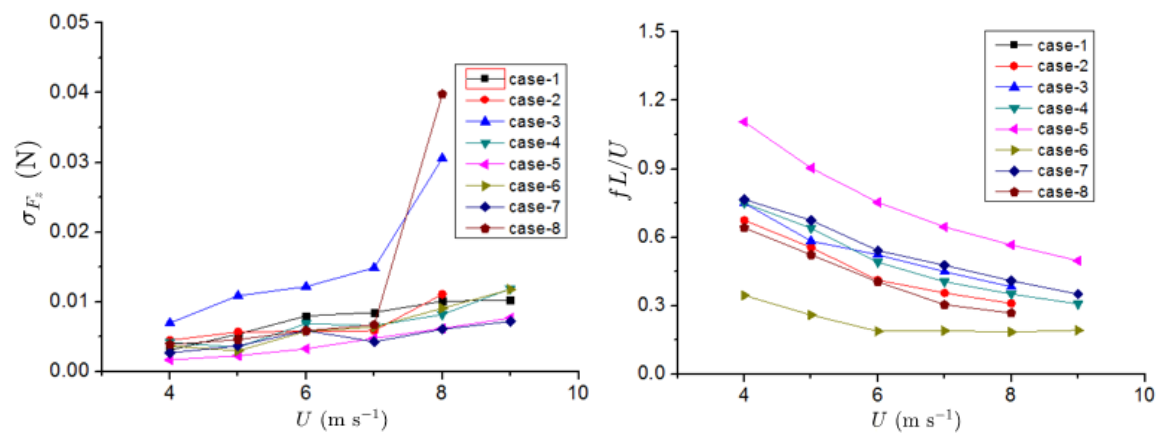

Figure 4. Left: Tangential force fluctuation for various incoming velocities for all leaf shapes. Right:

Non-dimensional tangential force-frequency.
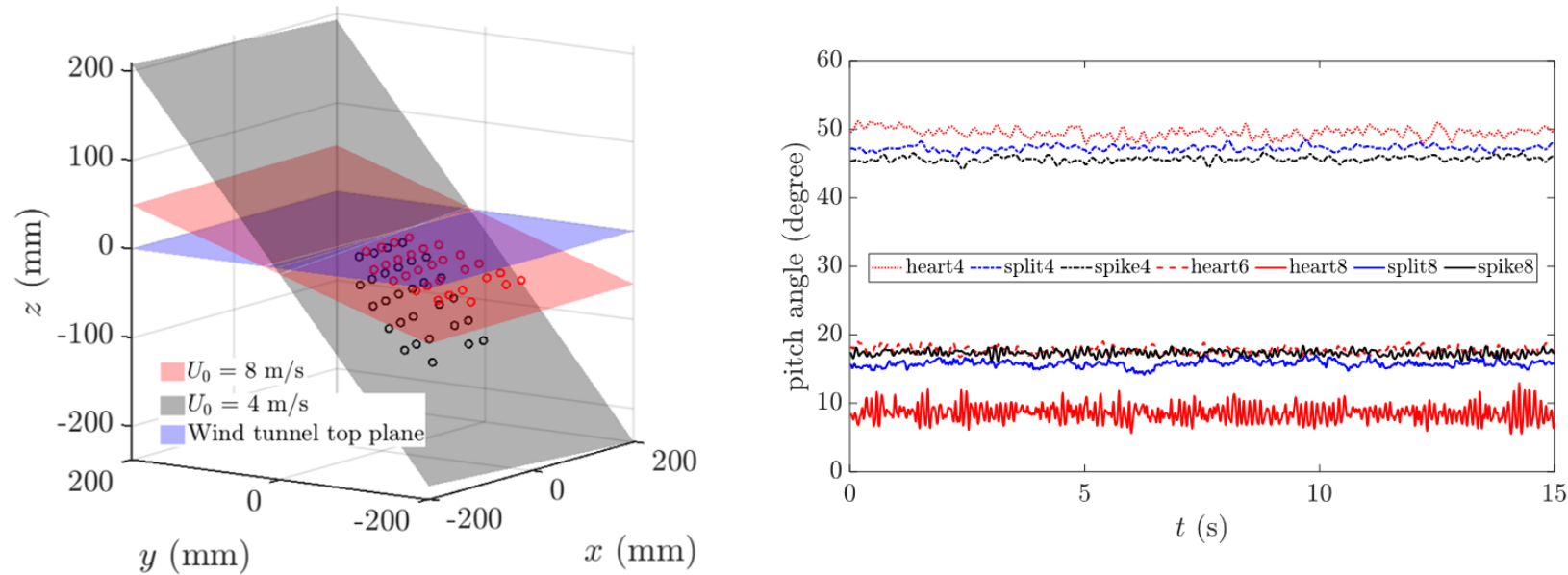

Figure 5. Left: Illustration of the leaf 2 mean deformation from DIC. Right: Pitch angle time series of leaf 2,3 and 8 at $U=4 \mathrm{~m} / \mathrm{s}$ and $8 \mathrm{~m} / \mathrm{s}$. 